QUT Digital Repository:

http://eprints.qut.edu.au/

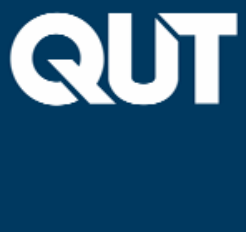

Aktarujjaman, M. and Haque, M. E. and Muttaqi, K. M. and Negnevitsky, Michael and Ledwich, Gerard F. (2007) Control stabilisation of multiple distributed generation. In Proceedings Australasian Universities Power Engineering Conference, 2007 (AUPEC 2007), pages pp. 636-640, Perth, Western Australia.

(C) Copyright 2007 IEEE

Personal use of this material is permitted. However, permission to reprint/republish this material for advertising or promotional purposes or for creating new collective works for resale or redistribution to servers or lists, or to reuse any copyrighted component of this work in other works must be obtained from the IEEE. 


\title{
Control Stabilisation of Multiple Distributed Generation
}

\author{
M. Aktarujjaman, Student Member, IEEE, M.E. Haque, Member, IEEE, K.M. Muttaqi, Senior \\ Member, IEEE, M. Negnevitsky, Senior Member, IEEE, and G. Ledwich, Senior Member, IEEE
}

\begin{abstract}
Grid connected distributed generation (DG) increases reliability and additional benefits for consumers as well as utilities. Recently, different types of DG are connected into distribution networks. Different control phenomena are applied based on types of DG. The stable and reliable operation of such power system requires sophisticated control. This paper presents a small-signal analysis for investigating dynamic behaviours of the system with multiple DG and also investigating control interactions between different types of DG. Dynamic model of a synchronous generator (SG) based hydro generator and inverter based photovoltaic/ wind distributed resources are considered for case studies to investigate grid connected and islanding mode of operation.
\end{abstract}

Index Terms-Multiple DG, Small-signal Analysis, Islanding Operation, Eigen value.

\section{INTRODUCTION}

Different strategies are applied to connect different distributed generation into the distribution systems as some are not suitable for connecting directly due to the nature of the energy produced. Conventional generators, such as hydro/diesel, can be connected directly to the distribution system. However, distributed generations such as wind, fuel cell, photo-voltaic and micro-turbine require power electronics interfaces grid integration. Synchronous generator (SG) presents inherent operating characteristics during frequency/speed and voltage/reactive power deviations. The control of frequency and voltage can be controlled by adjusting governor and exciter of the system. Inverter interfaced DG do not present natural connection between frequency and active power, neither between voltage and reactive power like $S G$ based DG. To achieve a stable operation with multiple DG, operations of control system must be coordinated [1-3].

During a large disturbance, the distributed generation with local loads may be separated from the utility system. This paper has investigated the stability of islanding operation during and subsequent islanding process with multiple DGs operated in a distribution system. Analytical approaches of frequency and voltage droop characteristic for $S G$ and frequency restoration method for inverter interfaced DG source are presented.

This paper is organized as follow, System description and frequency droop control and restoration methods are described in section 2. In section 3, control strategies of both generators in grid connected and islanded mode are discussed. Section 4 presents the results and discussions of the system dynamic behavior. Finally, conclusions are made in section 5 .

\section{SySTEM CONFIGURATIONS AND MODELING}

System configuration for a distribution system with multiple DG is shown in Fig.1. DG1 is a synchronous generator based mini hydro, which is equipped with governor and exciter control system. DG2 is inverter interfaced photovoltaic system with inverter control system. The control response of DG1 is relatively slower than that of DG2 control response during transients and small-signal dynamics. The multiple DGs are connected to the $110-\mathrm{kV} / 22-\mathrm{kV}$ substation as the point of common coupling with the utility grid $(110-\mathrm{kV})$. When the distribution system is connected with the utility grid, loads receive power both from the grid and from local DGs. If the grid power is lost because of IEEE 1547 events, faults, blackouts etc, the island operation will be automatically activated.

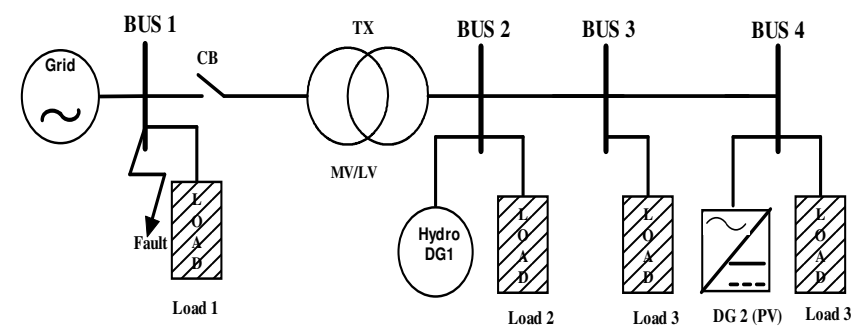

Fig.1: Single line diagram for a distribution system with multiple DG.

\section{A. Synchronous Generator (DG1):}

To examine the system frequency of DG1, the electromechanical equation for a generator per unit with closed loop control can be expressed as [5],

$$
2 H s \Delta f=\Delta P_{t}-\Delta P_{d}-D \Delta f
$$

Where, $H=$ inertial constant of overall turbine generator set in sec, $D=$ load damping constant, which is the ratio of load variation to frequency variation, and measured in p.u. megawatts per hertz, $s=$ Laplace operator, $\Delta P_{t}=$ change in mechanical power of turbine-generator set which is controlled 
by the governor system, $\Delta P_{d}=$ variation in the power demand due to the change in the load demand and/or the power mismatch introduced by the transformation from grid connected mode to islanded mode of operation. A simplified turbine-governor transfer function that represents the relation ship between the $\Delta P_{t}$ and $\Delta f$ is given by $[5,6]$ :

$$
\Delta P_{t}=-\frac{k_{1}}{k_{2}+s} \Delta f
$$

Where, $k_{1}$ and $k_{2}$ are constants related to the turbine time constant $\left(\tau_{g}\right)$ and the governor droop coefficient $(D)$ respectively and can be defined as,

$$
\begin{aligned}
& k_{1}=\frac{1}{D \tau_{g}} \\
& k_{2}=\frac{1}{\tau_{g}}
\end{aligned}
$$

The value of $\Delta f$ in the equation (1) represents the amount of frequency variation as a consequence of loss of generation or separation from the network which can be calculated as below,

$$
\Delta f=f_{0}-f_{L}
$$

Where $f_{L}$ is the nominal frequency and $f_{0}$ is the operating frequency. This frequency variation can be represented by the term of power imbalance in the islanded system as given below,

$$
\Delta f=\frac{K_{p}}{1+s T_{p}}\left(\Delta P_{t}-\Delta P_{d}\right)
$$

The per unit swing equation of DG1 given in eq. (1) can be combined with the turbine governor transfer function given in eq. (2) as follows,

$$
\begin{aligned}
& 2 H s \Delta f=-\frac{k_{1}}{k_{2}+s} \Delta f-\Delta P_{d}-D \Delta f \\
& 2 H s \Delta f+\frac{k_{1}}{k_{2}+s} \Delta f+D \Delta f=-\Delta P_{m i s} \\
& \left(2 H s+\frac{k_{1}}{k_{2}+s}+D\right) \Delta f=-\Delta P_{m i s}
\end{aligned}
$$

Where, $\Delta P_{d}=\Delta P_{m i s}$ represents the power mismatch. Hence, the transfer function model of the distribution network can be expressed as;

$$
\left.2 H s+\frac{k_{1}}{k_{2}+s}+D\right) \Delta f=-\Delta P_{m i s}
$$

Eq. (10) can be used for determining the rate and range of the frequency change of DG1 for a given value of real power mismatch during and subsequent islanding process and/or continuous local load variations.

The output frequency $f$ and voltage $v$ are controlled by employing active power-frequency droop characteristic and reactive power-voltage droop characteristic, shown in Fig.2 (a) and (b) respectively, to keep the system frequency and voltage within the limits. The frequency and voltage droop characteristic can be mathematically represented as [4]:

$$
\begin{aligned}
& f_{L}=f_{o}-k_{p} P \\
& V_{L}=V_{o}-k_{q} Q
\end{aligned}
$$

Where, $P$ and $Q$ are the real and reactive power outputs of the DG, $k_{p}$ and $k_{q}$ are the frequency and voltage droop slopes respectively, and $f_{L}$ and $V_{L}$ are the nominal values of the frequency and voltage, respectively. The characteristics shown in the Fig. 2 are steady state characterizes. They have a fixed slope in the region where the unit is operating within its power range. The droop is the locus where the steady state points are constrained to come to rest, but during dynamics the trajectory will deviate from the characteristics.

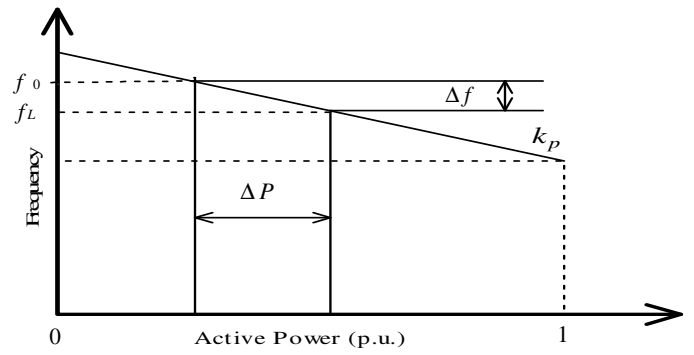

(a) frequency-active power $(f-P)$

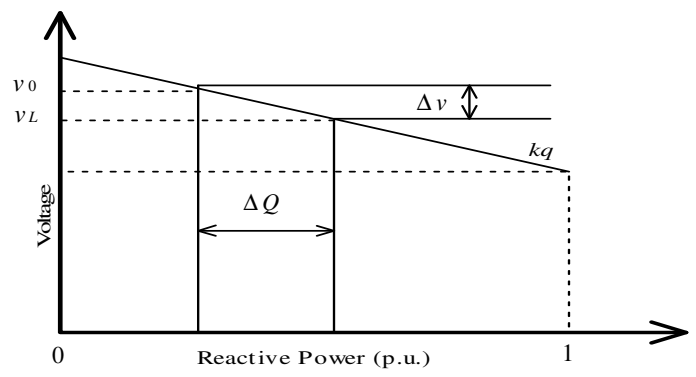

(b) voltage-reactive power $(V-Q)$

Fig.2: Droop characteristics.

In order to ensure the control law defined by (11) and (12), the droop gains can be calculated using (13) and (14) for the given range of frequency and voltage magnitude as follows,

$$
\begin{aligned}
& k_{q}=\frac{f_{o}-f_{L}}{P} \\
& k_{q}=\frac{V_{o}-V_{L}}{Q}
\end{aligned}
$$

Inverter based DG attempts to restore the frequency to its reference point by changing the output power as a function of change in frequency. This can be represented as,

$$
f_{L}=f_{o}-\frac{k s}{k_{f p} s+k_{f i}} P
$$

Where, $k$ is the droop co-efficient, and $k_{f p}$ and $k_{f i}$ are the frequency controller parameters.

The output of the inverter based DG can be expressed by frequency variation with controller parameters as, 


$$
\Delta P_{D G 2}=\frac{k_{f p} s+k_{f i}}{s} \Delta f
$$

Eq. (16) represents the power supplied by the DG2 in response to frequency/voltage disturbance. The output voltage $v$ is controlled by applying voltage droop method as given in eq. (11).

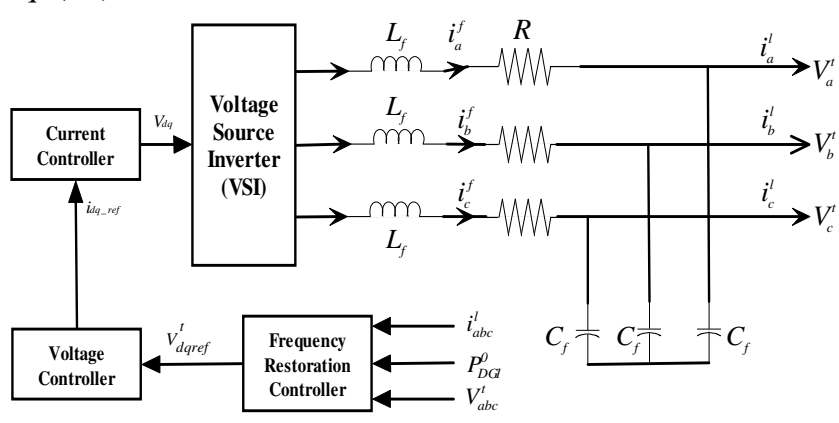

Fig.3: Inverter based DG block diagram

Fig. 3 shows the controller block diagram of a voltage source inverter (VSI) based DG. The control system consists of three main parts. First part is the frequency restoration control unit which provide the reference for voltage control unit. This controller sets the magnitude and frequency for the fundamental component of the inverter output voltage by applying frequency restoration law [7], which is provided in equation (15). The basic idea behind the frequency restoration method to mimic the isochronous governor characteristics of the synchronous generator into the inverter control system.

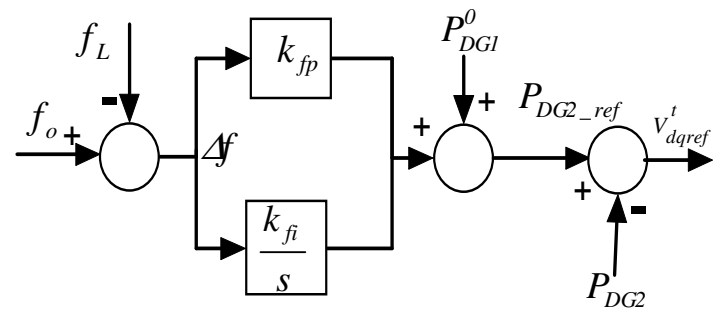

Fig.4: Frequency Restoration Controller

Fig.4 shows the frequency restoration controller block diagram. The real power reference is composed of power frequency variation $\Delta f$ and power supplied by DG1. The output of this control system is the reference for voltage controller loop of the DG2.

Fig. 5 shows the second and third parts of the VSI control system which are voltage and current controller. Voltage controller takes the reference from power controller of the system.

Local load change is another control issue for an islanded operation. Transferring from grid to islanded system creates initial real and reactive power imbalances in the islanded system. Furthermore, the voltage at the point of common coupling (PCC) is very sensitive to load changes in weak or islanded systems. The real power variation of the load is a function of voltage and frequency change and can be expressed as [5]:

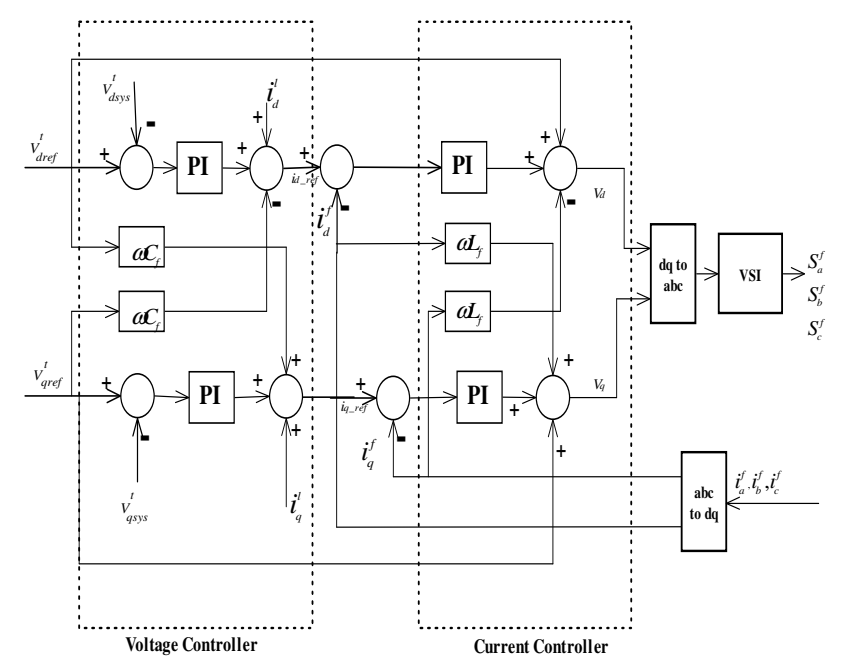

Fig.5: Voltage and current controller of VSI based DG

$$
\Delta P_{l}=\frac{\partial P_{l}}{\partial V} \Delta V+\frac{\partial P_{l}}{\partial f} \Delta f
$$

Eq.(17) can also be represented as,

$$
\Delta P_{l}=D_{v} \Delta V+D_{f} \Delta f
$$

Where, $D_{v}$ and $D_{f}$ are load-voltage and load-frequency sensitivity factor, respectively. A sudden change in real power can change of the load voltage. The changes in the load voltage $(\Delta V)$ can be expressed as a linear function of frequency changes as [7].

$$
\Delta V=k_{v} \Delta f
$$

Substituting the value of $\Delta V$ into eq.18, we get

$$
\Delta P_{l}=D_{v} k_{v} \Delta f+D_{f} \Delta f
$$

Eq. (20) can be rewritten as,

$$
\begin{aligned}
& \Delta P_{l}=k_{l} \Delta f \\
& \text { Where }, k_{l}=D_{v} k_{v}+D_{f}
\end{aligned}
$$

\section{CONTROL StRategIES OF MUltiPle DGS}

Control strategies are required to be developed for operation of i) grid-connected mode ii) islanding mode. In the grid connected mode, both DGs are utilized for supplying prespecified power to minimize the power import from the grid.

During grid connected mode of operation, systems frequency and voltage are controlled by the utility grid. DGs are supporting the load and providing support to the utility system.

In the case of islanding situation, both DG supplies its maximum available power to meet the load demand. If the power imbalance leads to under-generation situation, the system must apply load shedding policy. The dynamics of the system are changing rapidly in the case of islanding situations. A flexible control strategy is required to be developed to handle this dynamics [2].

Control system of inverter interfaced DG consists three control loops which are 1) frequency restoration control loop 
2) outer or voltage control loop and 3) inner or current control loop. Outer loop creates reference for inner loop. Inner control loop provides active damping for possible oscillations between the output filters of the inverters and the transmission lines [4].

Control stabilization of the system with multiple DG may creat poor power qualities that may lead to voltage and angle instability.

The real power shearing between the inverter and synchronous generator is obtain by introducing droop characteristics as given in eq. (11). The frequency $f_{L}$ is set according to the droop gain $\left(k_{p}\right)$ and the magnitude of the gain can be found by using eq. (13). Fast frequency restoration is achieved by introducing restoration method as eq. (15).

When the system is disconnected, it is given by operated with DG1, DG2 and local load. The total electrical load of the system is seen by the DG1 can be expressed as [7],

$$
\Delta P_{d}=\Delta P_{l}+\Delta P_{D G 2}+\Delta P_{m i s}
$$

Where $\Delta P_{l}, \Delta P_{D G 2}$ and $\Delta P_{m i s}$ are the load variations, DG2 power supply and power mismatch after disconnecting from the utility grid, respectively.

Substituting eq. (16) and (21) into eq. (22) we obtain,

$$
\Delta P_{d}=k_{l} \Delta f+\frac{k_{f p} s+k_{f i}}{s} \Delta f+\Delta P_{m i s}
$$

By substituting eqs. (23) and (2) into eq. (1), we obtain the transfer function of the system between frequency changes to power mismatch as follows,

$$
\frac{\Delta f}{\Delta P_{m i s}}=\left(\frac{-1}{2 H s}\right) \frac{s^{2}+s k_{2}}{s^{3}+s^{2} x_{1}+s x_{2}+x_{3}}
$$

$$
x_{1}=\left(k_{2}+\frac{k_{f p}+k_{l}}{2 H}\right), x_{2}=\left(\frac{k_{f i}+k_{1}}{2 H}\right), x_{3}=\left(\frac{k_{2}\left(k_{f i}+k_{l}\right)}{2 H}\right)
$$

The denominator of the transfer function provides the information about the desired pole for the closed loop controller. Appropriate selection of theses parameters determine the desired frequency response characteristic of the islanded system for a given power mismatch.

\section{RESULTS AND DISCUSSIONS}

Simulations were carried out on the system shown in Fig. 1 and using the transfer function stated in eq. (24). The parameters are presented in appendix-A.

Fig.6 shows the step response of the system during $20 \%$ power mismatch. Power mismatch due to the disconnection from utility grid or local load variations is considered. Cocoordinated control system of DGs compensates the power imbalance and helps to back to normal operating mode.

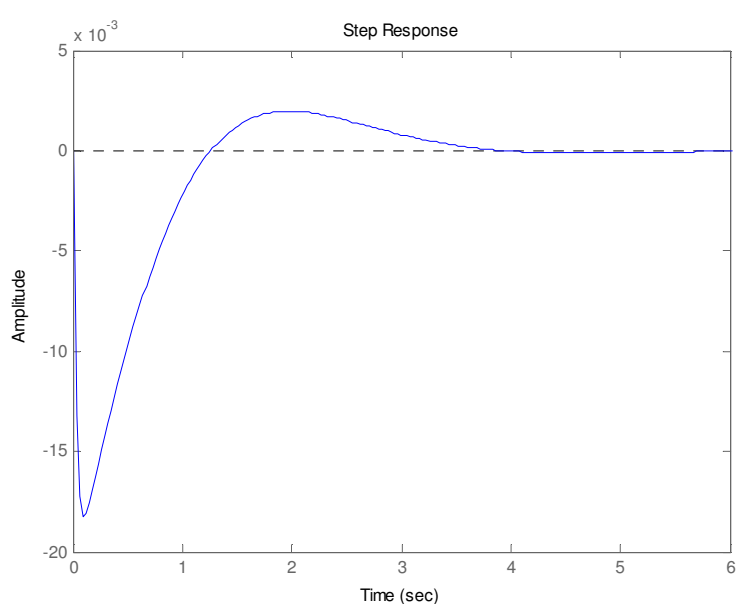

Fig.6: Step response of the power mismatch

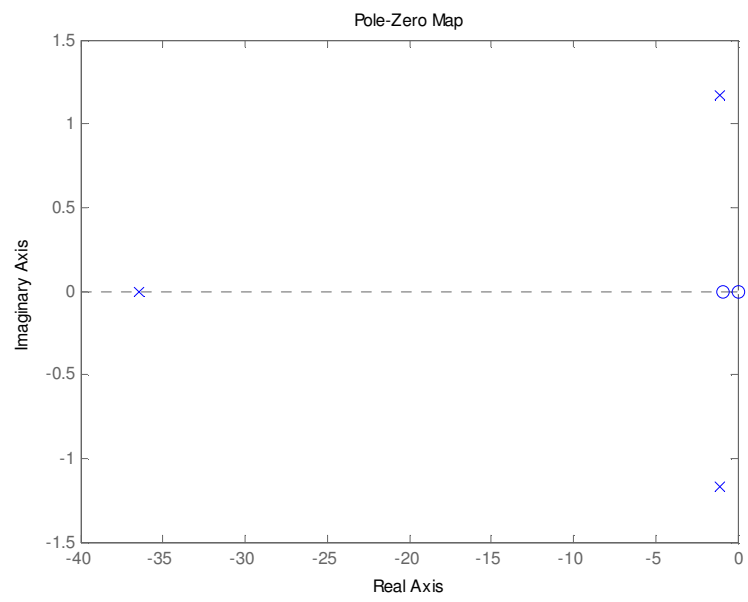

Fig.7: Pole-zero location of the system in s-plane

The system doesn't have any zero eigen-value. Only the non-zero eigen-values of the system are important for system stability studies $[5,6]$. The system presents three negative real eigen-values as given in Table-I and, consequently, it has a stable damped response.

Fig. 7 shows the zero and pole locations for system parameters given in appendix A and power mismatch.

TABLE-I

SYSTEM EIGEN VALUE

\begin{tabular}{|c|c|c|}
\hline EIGEN-VALUE & DAMPING & FREQ. (RAD/S) \\
\hline$-1.06 \mathrm{E} 0+1.17 \mathrm{E}+0 \mathrm{I}$ & $6.69 \mathrm{E}-001$ & $1.58 \mathrm{E}+000$ \\
\hline$-1.06 \mathrm{E} 0-1.17 \mathrm{E}+0 \mathrm{I}$ & $6.69 \mathrm{E}-001$ & $1.58 \mathrm{E}+000$ \\
\hline$-3.64 \mathrm{E}+1$ & $1.00 \mathrm{E}+000$ & $3.64 \mathrm{E}+001$ \\
\hline
\end{tabular}




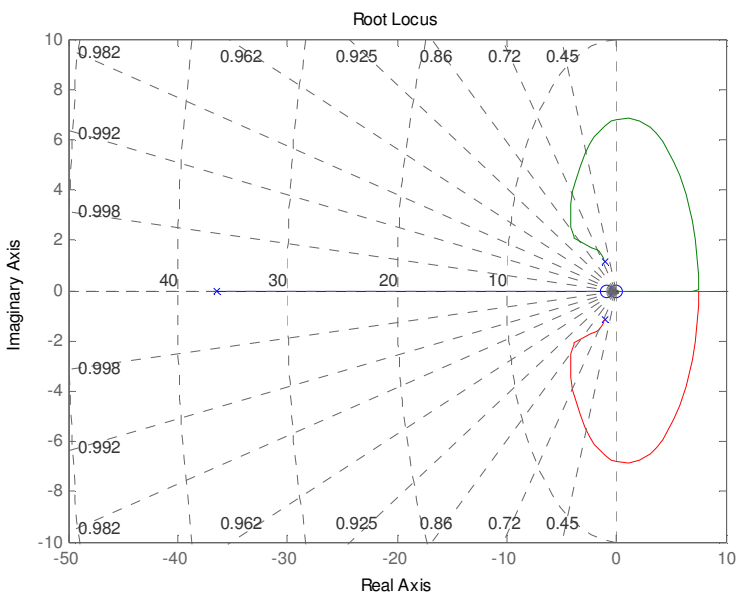

Fig.8: Root locus for islanded system with $20 \%$ power mismatch.

Fig. 8 shows the root locus for the system as a function of power mismatch. The system response shows that the stability can be achieved during $20 \%$ power mismatch.

\section{CONCLUSIONS}

This paper presents a strategy for control stabilization of multiple DG. A dynamic model of multiple-DG system is developed to investigate the stable operating condition of the DGs. The impact of multiple DG in the distribution systems during islanded situations is considered. Dynamic response of the system confirms that stable operation of the system with multiple DG is possible during and subsequent islanding period. It is observed that initial power mismatch due to islanding and local load variations can be overcome by applying frequency restoration into DG2 and frequency droop control in DG1. System stability can be achieved by appropriate control strategy developed for DGs.

\section{APPENDIX}

Following control parameters for DG1 and DG2 are used for studying the system stability.

\begin{tabular}{|l|l|c|c|}
\hline \multicolumn{2}{|c|}{ DG1 (3.5 MVA) } & \multicolumn{2}{c|}{ DG2 (0.75 MVA) } \\
\hline $\mathrm{D}$ & 0.05 & $k_{p}$ & 0.2 \\
\hline$\tau_{g}$ & 1.1 & $k_{i}$ & 83.3 \\
\hline $\mathrm{H}$ & $1.6 \mathrm{~s}$ & $\Delta P_{d}$ & $20 \%$ \\
\hline
\end{tabular}

\section{REFERENCES}

[1] N. Pogaku, M. Prodanovic and Timothy C. Green, "Modeling Analysis and Testing of Autonomous Operation of an Inverter-Based Microgrid", IEEE Trans. on Power Electronics, Vol. 22, No. 2, March 2007, pp. 613-624.

[2] F. Katiraei and M.R. Iravani, "Power Management Strategies for a Microgrid With Multiple Distributed Generation Units", IEEE Trans. on Power Systems, Vol. 21, No. 4, November 2006, pp. 1821-1831.

[3] J.A. Lopes, C.L. Moreira and A.G. Madureira,“ Defining Control Strategies for Microgrids Islanded Operation”, IEEE Transactions on Power Systems, Vol. 21, No.2, May 2006, pp. 916-924.
[4] E.A.A. Coelho, P.C. Cortizo, and P.F.D. Garcia, "Small-Signal Stability for Parallel-Connected Inverters in Stand-Alone AC Supply Systems", IEEE Trans. on Industry Applications, Vol. 38, No. 2, March/April 2002, pp. 533-542.

[5] P. Kundur, "Power system stability and control", New York, McGrawHill, 1994.

[6] P.M. Anderson, A.A. Fouad, "Power system control and stability", The Iowa State University Press, 1977.

[7] F. Katiraei and C. Abbey, "Design of an Electronically-Interfaced Dispatchable Power Generation Source to Facilitate Islanding and Autonomous Operation of a Distributed Network" 4th Power Conversion Conference (PCC2007) Nagoya-Japan, April 2007.

\section{BIOGRAPHIES}

Md. Aktarujjaman (M'00) received the B.Sc.TE. from the Islamic University of Technology, Bangladesh, in 2000. He obtained Masters in Information Technology from Charles Sturt University, Australia in 2002. He is currently pursuing the Ph.D degree at the University of Tasmania. His special fields of interests are renewable energy, distributed generation, power system control and protection.

Md. Enamul Haque (IEEE Student M'97, M'03) was born in Bangladesh in 1970. He graduated in electrical and electronic engineering from Bangladesh Institute of Technology (BIT), Rajshahi, Bangladesh, in 1995. He received the M.Engg. degree in electrical engineering from University Technology Malaysia in 1998, and Ph.D. degree in electrical engineering from The University of New South Wales, Sydney, Australia, in 2002. He has worked as an Assistant Professor for King Saud University, Saudi Arabia and United Arab Emirates University, UAE for four years. Dr. Haque is currently working as a Research Fellow in the school of Engineering of University of Tasmania, Australia. His research interests include power electronics and DSP based electric drives, Wind/Solar remote area power supply system, EMC/EMI issues in power electronics and drives, and Renewable energy applications.

K. M. Muttaqi (A'00-SM'05) received the Ph.D. degree from Multimedia University, Malaysia, in 2001. Currently, he is a Senior Lecturer at the School of Engineering, University of Tasmania, Australia. He was associated with the Queensland University of Technology, Australia as a Postdoctoral Research Fellow from 2000 to 2002. Previously, he also worked for Multimedia University as a Lecturer for three years. His special fields of interests include distributed generation, renewable energy, distribution system automation, power system planning, and artificial intelligence. He has published more than 50 technical papers in these areas.

Michael Negnevitsky (M'95) received the B.S.E.E. (Hons.) and Ph.D. degrees from Byelorussian University of Technology, Minsk, Belarus, in 1978 and 1983, respectively. Currently, he is an Associate Professor in the School of Engineering at the University of Tasmania, Hobart, Australia. From 1984 to 1991, he was a Senior Research Fellow and Senior Lecturer in the Department of Electrical Engineering, Byelorussian University of Technology. After arriving in Australia, he was with Monash University, Melbourne, Australia. His interests are power system analysis, power quality, and intelligent systems applications in power systems. Dr. Negnevitsky is a Chartered Professional Engineer, a Senior Member of the Institution of Engineers Australia, and a Member of CIGRE AP36 (Electromagnetic Compatibility), Australian Technical Committee.

Gerard Ledwich (M'73-SM'92) received the Ph.D. in electrical engineering from the University of Newcastle, Australia, in 1976. He has been Chair Professor in Electrical Asset Management at Queensland University of Technology, Australia, since 1998. He was Head of electrical engineering at the University of Newcastle from 1997 to 1998. Previously, he was associated with the University of Queensland from 1976 to 1994. His interests are in the areas of power systems, power electronics, and controls. Prof. Ledwich is a Fellow of the Institution of Engineers Australia. 